\title{
Higgs boson production via gluon fusion: Soft-gluon resummation including mass effects
}

\author{
Timo Schmidt ${ }^{1,2, *}$ and Michael Spira ${ }^{1}$ \\ ${ }^{1}$ Paul Scherrer Institut, CH-5232 Villigen PSI, Switzerland \\ ${ }^{2}$ Physik Institut, Zürich University, CH-8057 Zürich, Switzerland \\ (Received 11 September 2015; published 22 January 2016)
}

\begin{abstract}
We analyze soft and collinear gluon resummation effects at the $\mathrm{N}^{3} \mathrm{LL}$ level for Standard Model Higgs boson production via gluon fusion $g g \rightarrow H$ and the neutral scalar and pseudoscalar Higgs bosons of the minimal supersymmetric extension at the next-to-next-to-next-to-leading-log ( $\left.\mathrm{N}^{3} \mathrm{LL}\right)$ and next-to-next-toleading-log (NNLL) level, respectively. We introduce refinements in the treatment of quark mass effects and subleading collinear gluon effects within the resummation. Soft and collinear gluon resummation effects amount to up to about $5 \%$ beyond the fixed-order results for scalar and pseudoscalar Higgs boson production.
\end{abstract}

DOI: 10.1103/PhysRevD.93.014022

\section{INTRODUCTION}

The Standard Model (SM) of elementary particle physics has been established as a very successful theory that describes the properties and strong, weak and electromagnetic interactions of all known elementary particles [1]. The recent discovery of the Higgs boson with a mass of about $125 \mathrm{GeV}$ [2] completed the particle content of the SM and established the Higgs mechanism for electroweak symmetry breaking [3]. Its couplings to SM gauge bosons, i.e. to $Z Z$, $W^{+} W^{-}$, and fermion pairs ( $\tau$ leptons and bottom quarks) as well as the loop-induced couplings to gluon and photon pairs, have been measured with accuracies of $10 \%-50 \%$. All measured couplings are in agreement with the SM predictions within their uncertainties [4]. In addition there are very strong indications that the newly discovered boson carries zero spin and positive $\mathcal{C} \mathcal{P}$-parity [5]. Its discovery is of vital importance for the mathematical consistency of the SM and the success of the predictions for the precision electroweak observables which are in striking agreement with measurements at LEP and SLC [6]. Based on the present situation it is highly relevant to test the properties of the discovered particle in more detail. The measured inclusive production and decay rates are in agreement with the theoretical predictions within the corresponding uncertainties.

At hadron colliders such as the LHC neutral Higgs bosons of the Standard Model are copiously produced by the gluon fusion process $g g \rightarrow H$, which is mediated by top and to a lesser extent bottom and charm quark loops. Due to the large top Yukawa coupling and the large gluon luminosities gluon fusion comprises the dominant Higgs boson production mechanism for the SM [7].

In the past the next-to-leading-order (NLO) QCD corrections to the top and bottom/charm quark loops have been calculated [8-11]. They increase the cross

*Present address: Albert-Ludwigs-Universität Freiburg, Physikalisches Institut, D-79104 Freiburg, Germany. sections by up to $90 \%$. The full quark and Higgs mass dependencies for the quark loops have been included [9-11]. The scale dependence decreased from $\mathcal{O}(100 \%)$ to $\sim 20 \%$ at NLO. The NLO results indicated that the limit of heavy top quarks provides a reliable approximation of the full relative QCD corrections within $~ 5 \%$ for a Higgs mass of $125 \mathrm{GeV}[9,12]$. In this limit the cross section factorizes into a part originating from an effective Lagrangian derived by integrating out the top quark supplemented by gluonic and light quark corrections within the effective low-energy theory $[8,13]$. Within this approach the next-to-next-to-leading-order (NNLO) QCD corrections have been calculated leading to a further increase of the cross section by $20 \%-30 \%$ [14-16] and a further reduction of the scale dependence to $\sim 10 \%$. Mass effects beyond the heavy top approximation have been studied at NNLO in a heavy top mass expansion [17]. These mass effects amount to less than a percent in the relative QCD corrections below the $t \bar{t}$-threshold. The NNLO results have been improved by a soft and collinear gluon resummation at the NNLL level $[18,19]$ that add another 5\%-10\% beyond NNLO to the total Higgs production cross section. In addition the small quark mass effects have been included in the soft-gluonresummed result at next-to-leading-log (NLL) [20]. Very recently the next-to-next-to-next-to-leading-order $\left(\mathrm{N}^{3} \mathrm{LO}\right)$ corrections have been computed in the limit of heavy top quarks first approximately deriving the leading terms in a threshold expansion [21] and then completely $[22,23]$. These increase the cross section by only a few percent and reduce the scale dependence to a level of about $5 \%$. They coincide with the soft + collinear gluon approximation at $\mathrm{N}^{3} \mathrm{LO}$ at the level of a few percent [24-26]. This signals that soft gluon effects are less important at $\mathrm{N}^{3} \mathrm{LL}$ than at the NNLL level, thus underlining a proper perturbative reliability of the $\mathrm{N}^{3} \mathrm{LO}$ and $\mathrm{N}^{3} \mathrm{LL}$ results. These results have been completed by the calculation of the full electroweak corrections at NLO 
[27] and beyond [28] that provide a further increase of the cross section by about $5 \%$.

One of the most attractive extensions of the SM is the minimal supersymmetric SM (MSSM) that requires the introduction of two Higgs doublets and thus predicts the existence of five elementary Higgs bosons; two neutral $C P$-even $h, H$; one neutral $C P$-odd $A$; and two charged ones $H^{ \pm}$[29]. At LO the Higgs sector is described by two independent input parameters that in the case of a real MSSM are usually chosen to be the pseudoscalar Higgs mass $M_{A}$ and the parameter $\operatorname{tg} \beta$ defined as the ratio of the two $C P$-even vacuum expectation values. Higher-order corrections to the MSSM Higgs masses and couplings turned out to be large with the dominant piece originating from contributions induced by the large top Yukawa coupling [30]. Including all relevant corrections up to the three-loop level the mass of the light scalar Higgs boson $h$ is bound to be smaller than $\sim 135 \mathrm{GeV}$ [31]. The light scalar Higgs boson becomes SM-Higgs-like close to its upper mass bound, thus allowing for the possibility of the Higgs boson found at the LHC to be identified with the light scalar $h$. Global fits to the MSSM leave a small region within the MSSM parameter space where the discovered Higgs particle could also be the heavy scalar $H$ [32] but this region is disfavored by LHC data [33]. The Higgs couplings to intermediate gauge bosons and fermions are modified by additional factors shown in Table I that only depend on the mixing angles $\alpha$ and $\beta$ where $\alpha$ denotes the mixing angle between the neutral $C P$-even Higgs states. For large values of $\operatorname{tg} \beta$ the down-type Yukawa couplings are strongly enhanced, while the up-type Yukawa couplings are suppressed, unless the light (heavy) scalar Higgs mass ranges at its upper (lower) bound, where the couplings become SM sized.

The dominant Higgs boson production mechanisms for small and moderate values of $\operatorname{tg} \beta$ are the gluon fusion processes $g g \rightarrow h, H, A$ that are mediated by top and bottom and in addition top-squark and sbottom loops in the case of the scalar Higgs bosons $h, H$. For large $\operatorname{tg} \beta$ the leading role is taken over by Higgs radiation off bottom quarks due to the strongly enhanced bottom Yukawa couplings [34]. In the past the NLO QCD corrections for pseudoscalar Higgs boson production have been derived in the limit of heavy top quarks [35] and later including the full Higgs and quark mass dependencies $[9,36]$. They are large ranging up to about $100 \%$. In the

TABLE I. Higgs couplings in the MSSM to fermions and gauge bosons $[V=W, Z]$ relative to the SM couplings.

\begin{tabular}{lcccc}
\hline \hline$\phi$ & & $g_{u}^{\phi}$ & $g_{d}^{\phi}$ & $g_{V}^{\phi}$ \\
\hline SM & $H$ & 1 & 1 & 1 \\
MSSM & $h$ & $\cos \alpha / \sin \beta$ & $-\sin \alpha / \cos \beta$ & $\sin (\beta-\alpha)$ \\
& $H$ & $\sin \alpha / \sin \beta$ & $\cos \alpha / \cos \beta$ & $\cos (\beta-\alpha)$ \\
& $A$ & $1 / \operatorname{tg} \beta$ & $\operatorname{tg} \beta$ & 0 \\
\hline \hline
\end{tabular}

limit of heavy top quarks the calculation of the SM Higgs boson at NNLO QCD has been extended to the pseudoscalar case, too [16,37], inducing a moderate increase of the total cross section by about $20 \%-30 \%$ as in the SM Higgs case. However, the limit of heavy top quarks is only applicable for the pure top loop contributions so that for bottom-loop dominance for large values of $\operatorname{tg} \beta$ we are left with NLO accuracy. For the case of top-loop dominance, i.e. for small and moderate values of $\operatorname{tg} \beta$ within the MSSM, the soft-gluon resummation has been extended to the NNLL level for the pseudoscalar Higgs boson [38] resulting in corrections of $\mathcal{O}(10 \%)$ beyond NNLO. For the light and heavy scalar MSSM Higgs bosons the NLO QCD corrections to the squark loops have been calculated in the approximate limit of heavy squarks [39] yielding NLO corrections close to $100 \%$ as for the quark loops. The nextto-leading order computation for the squark loops including the full mass dependencies was performed later $[11,40]$ for the pure QCD corrections. The genuine supersymmetric QCD corrections have been derived in Ref. [41] for large supersymmetric particle masses; i.e. the full Higgs mass dependence has not been taken into account. For gluino masses much larger than the top-squark and top masses the results of [41] develop a logarithmic singularity in the gluino mass that seems to contradict the AppelquistCarazzone decoupling theorem [42] at first glance. This problem has been solved by a detailed renormalization group analysis with the corresponding decoupling techniques for heavy particles [43]. The full supersymmetric QCD corrections including all mass dependencies have been completed in Refs. [44]. The genuine SUSY-QCD corrections at NLO are large, too, modifying the total gluonfusion cross sections by up to $\sim 100 \%$ depending on the MSSM parameters of the Higgs and top-squark/sbottom sectors.

In this work we will present the soft + virtual + collinear gluon resummed results at $\mathrm{N}^{3} \mathrm{LL}$ for scalar Higgs boson production via gluon fusion including a consistent treatment of top and bottom mass effects with an extension to the inclusion of additional subleading collinear gluon effects. For the pseudoscalar Higgs boson we will present the analogous results up to the NNLL level, since the fixedorder $\mathrm{N}^{3} \mathrm{LO}$ result does not exist for this case. The paper is organized as follows. In Sec. II we will describe our method for the scalar and pseudoscalar Higgs bosons of the SM and MSSM. In Sec. III we will present results and discuss the comparison with previous calculations. In Sec. IV we will finally present our conclusions.

\section{SM HIGGS BOSON PRODUCTION}

Higgs boson production via gluon fusion is mediated by top and to a lesser extent bottom and charm triangle loops at LO. Following the notation of Ref. [18] the production cross section of scalar Higgs boson production via gluon fusion $g g \rightarrow H$ can be cast into the form 


$$
\begin{aligned}
\sigma\left(s, M_{H}^{2}\right)= & \sigma_{0} \sum_{i j} \int_{\tau_{H}}^{1} d \tau \frac{d \mathcal{L}^{i j}}{d \tau} \\
& \times \int_{0}^{1} d z z G_{i j}\left(z ; \alpha_{s}\left(\mu_{R}^{2}\right), \frac{M_{H}^{2}}{\mu_{R}^{2}} ; \frac{M_{H}^{2}}{\mu_{F}^{2}}\right) \delta\left(z-\frac{\tau_{H}}{\tau}\right) \\
\sigma_{0}= & \frac{G_{F}}{288 \sqrt{2} \pi}\left|\sum_{Q} A_{Q}\left(\tau_{Q}\right)\right|^{2} \\
A_{Q}(\tau)= & \frac{3}{2} \tau[1+(1-\tau) f(\tau)] \\
f(\tau)= & \begin{cases}\arcsin ^{2} \frac{1}{\sqrt{\tau}} & \tau \geq 1 \\
-\frac{1}{4}\left[\log \frac{1+\sqrt{1-\tau}}{1-\sqrt{1-\tau}}-i \pi\right]^{2} & \tau<1\end{cases}
\end{aligned}
$$

where the sum over $i, j$ runs over all contributing initial states, i.e. only gluons at LO. The term $\mathcal{L}^{i j}$ denotes the corresponding parton-parton luminosity, $M_{H}$ the Higgs boson mass, $\mu_{R / F}$ the renormalization/factorization scale, $\alpha_{s}$ the strong coupling constant and $G_{F}$ the Fermi constant. The variable $\tau_{Q}$ is defined as

$$
\tau_{Q}=4 \frac{m_{Q}^{2}}{M_{H}^{2}}
$$

where $m_{Q}$ is the corresponding loop quark mass. The lower integration bound is given by $\tau_{H}=M_{H}^{2} / s$. The integration kernel $G_{i j}$ can be computed as a perturbative expansion in the strong coupling constant,

$$
\begin{aligned}
& G_{i j}\left(z ; \alpha_{s}\left(\mu_{R}^{2}\right), \frac{M_{H}^{2}}{\mu_{R}^{2}} ; \frac{M_{H}^{2}}{\mu_{F}^{2}}\right) \\
& \quad=\alpha_{s}^{2}\left(\mu_{R}^{2}\right) \sum_{n=0}^{\infty}\left(\frac{\alpha_{s}\left(\mu_{R}^{2}\right)}{\pi}\right)^{n} G_{i j}^{(n)}\left(z ; \frac{M_{H}^{2}}{\mu_{R}^{2}} ; \frac{M_{H}^{2}}{\mu_{F}^{2}}\right)
\end{aligned}
$$

where the LO and NLO expressions are given by

$$
\begin{aligned}
G_{i j}^{(0)}(z)= & \delta_{i g} \delta_{j g}(1-z) \\
G_{g g}^{(1)}\left(z ; \frac{M_{H}^{2}}{\mu_{R}^{2}} ; \frac{M_{H}^{2}}{\mu_{F}^{2}}\right)= & \delta(1-z)\left\{c_{H}\left(\tau_{Q}\right)+6 \zeta_{2}+\frac{33-2 N_{F}}{6} \log \frac{\mu_{R}^{2}}{\mu_{F}^{2}}\right\}+12 \mathcal{D}_{1}(z) \\
& +6 \mathcal{D}_{0}(z) \log \frac{M_{H}^{2}}{\mu_{F}^{2}}+P_{g g}^{\mathrm{reg}}(z) \log \frac{(1-z)^{2} M_{H}^{2}}{z \mu_{F}^{2}}-6 \frac{\log z}{1-z}+d_{g g}^{H}\left(z, \tau_{Q}\right) \\
G_{g q}^{(1)}\left(z ; \frac{M_{H}^{2}}{\mu_{R}^{2}} ; \frac{M_{H}^{2}}{\mu_{F}^{2}}\right)= & \frac{1}{2} P_{g q}(z) \log \frac{(1-z)^{2} M_{H}^{2}}{z \mu_{F}^{2}}+d_{g q}^{H}\left(z, \tau_{Q}\right) \\
G_{q \bar{q}}^{(1)}\left(z ; \frac{M_{H}^{2}}{\mu_{R}^{2}} ; \frac{M_{H}^{2}}{\mu_{F}^{2}}\right)= & d_{q \bar{q}}^{H}\left(z, \tau_{Q}\right)
\end{aligned}
$$

with the NLO quark mass dependence contained in the functions $c_{H}$ and $d_{i j}^{H}(i j=g g, g q, q \bar{q})$. Explicit results for these functions can be found in [9]. ${ }^{1}$ In the limit of heavy top quarks they approach the following simple expressions,

$$
\begin{aligned}
c_{H}\left(\tau_{Q}\right) & \rightarrow \frac{11}{2} \\
d_{g g}^{H}\left(z, \tau_{Q}\right) & \rightarrow-\frac{11}{2} \frac{(1-z)^{3}}{z} \\
d_{g q}^{H}\left(z, \tau_{Q}\right) & \rightarrow \frac{2}{3} z-\frac{(1-z)^{2}}{z} \\
d_{q \bar{q}}^{H}\left(z, \tau_{Q}\right) & \rightarrow \frac{32}{27} \frac{(1-z)^{3}}{z} .
\end{aligned}
$$

\footnotetext{
${ }^{1}$ Note that the results of [9] have to be divided by $z$ due to our different normalization of the functions $d_{i j}^{H}\left(z, \tau_{Q}\right)$ $(i j=g g, g q, q \bar{q})$.
}

For the NLO results presented above we have used the notation $(i=0,1, \ldots)$

$$
\mathcal{D}_{i}(z)=\left(\frac{\log ^{i}(1-z)}{1-z}\right)_{+}
$$

for the plus distributions and $\zeta_{2}=\pi^{2} / 6$. The AltarelliParisi splitting kernels are given by [45]

$$
\begin{aligned}
P_{g g}(z) & =6 \mathcal{D}_{0}(z)+P_{g g}^{\mathrm{reg}}(z) \\
P_{g g}^{\mathrm{reg}}(z) & =6\left[\frac{1}{z}-2+z(1-z)\right] \\
P_{g q}(z) & =\frac{4}{3} \frac{1+(1-z)^{2}}{z} .
\end{aligned}
$$

The results of the NNLO pieces $G_{i j}^{(2)}$ of the coefficient function can be extracted from Refs. $[14,16]$ and the Mellin 
transforms of their leading soft + virtual contributions are given explicitly in Ref. [18].

\section{A. Soft and collinear gluon resummation}

In this work we will resum soft and collinear gluon effects up to all orders in the perturbative expansion. This will be performed systematically in Mellin space. The Mellin moment of the gluon-fusion cross section is defined as

$$
\tilde{\sigma}\left(N, M_{H}^{2}\right)=\int_{0}^{1} d \tau_{H} \tau_{H}^{N-1} \sigma\left(s, M_{H}^{2}\right)
$$

so that the moments acquire the factorized form

$$
\begin{aligned}
\tilde{\sigma}\left(N-1, M_{H}^{2}\right)= & \sigma_{0} \sum_{i j} \tilde{f}_{i}\left(N, \mu_{F}^{2}\right) \tilde{f}_{j}\left(N, \mu_{F}^{2}\right) \\
& \times \tilde{G}_{i j}\left(N ; \alpha_{s}\left(\mu_{R}^{2}\right), \frac{M_{H}^{2}}{\mu_{R}^{2}} ; \frac{M_{H}^{2}}{\mu_{F}^{2}}\right) .
\end{aligned}
$$

The Mellin transformation can be inverted by means of the contour integral

$$
\begin{aligned}
\sigma\left(s, M_{H}^{2}\right)= & \sigma_{0} \sum_{i j} \int_{C-i \infty}^{C+i \infty} \frac{d N}{2 \pi i}\left(\frac{M_{H}^{2}}{s}\right)^{-N+1} \tilde{f}_{i}\left(N, \mu_{F}^{2}\right) \\
& \times \tilde{f}_{j}\left(N, \mu_{F}^{2}\right) \tilde{G}_{i j}\left(N ; \alpha_{s}\left(\mu_{R}^{2}\right), \frac{M_{H}^{2}}{\mu_{R}^{2}} ; \frac{M_{H}^{2}}{\mu_{F}^{2}}\right)
\end{aligned}
$$

where the value of the offset $C$ has to be chosen such that all singularities of the $N$-moments are located on the left of the contour. Soft and collinear gluon effects arise in the limit $z \rightarrow 1$ that corresponds to the limit of large $N$ in Mellin space. The leading contributions in Mellin space only appear in the $g g$ initial state, while all other initial states are suppressed at $\mathcal{O}(1 / N)[18]$ as can be inferred explicitly from the NLO results presented in Eq. (4). ${ }^{2}$ Following Refs. $[18,46]$ the leading contributions to the coefficient function $\tilde{G}_{g g}$ in Mellin space are logarithmic in $N$ and follow the perturbative expansion

$$
\begin{aligned}
\tilde{G}_{g g}\left(N ; \alpha_{s}\left(\mu_{R}^{2}\right), \frac{M_{H}^{2}}{\mu_{R}^{2}} ; \frac{M_{H}^{2}}{\mu_{F}^{2}}\right) & =\alpha_{s}^{2}\left(\mu_{R}^{2}\right)\left\{1+\sum_{n=1}^{\infty}\left(\frac{\alpha_{s}\left(\mu_{R}^{2}\right)}{\pi}\right)^{n} \sum_{m=0}^{2 n} G_{H}^{(n, m)} \log ^{m} N\right\}+\mathcal{O}\left(\frac{1}{N}\right) \\
& =\tilde{G}_{g g}^{(\text {res })}\left(N ; \alpha_{s}\left(\mu_{R}^{2}\right), \frac{M_{H}^{2}}{\mu_{R}^{2}} ; \frac{M_{H}^{2}}{\mu_{F}^{2}}\right)+\mathcal{O}\left(\frac{1}{N}\right)
\end{aligned}
$$

where the second line indicates that the leading parts can be resummed in closed form. The all-order resummed expression can be cast into the form

$\tilde{G}_{g g}^{(\mathrm{res})}\left(N ; \alpha_{s}\left(\mu_{R}^{2}\right), \frac{M_{H}^{2}}{\mu_{R}^{2}} ; \frac{M_{H}^{2}}{\mu_{F}^{2}} ; \frac{M_{H}^{2}}{m_{t}^{2}}\right)=\alpha_{s}^{2}\left(\mu_{R}^{2}\right) C_{g g}\left(\alpha_{s}\left(\mu_{R}^{2}\right), \frac{M_{H}^{2}}{\mu_{R}^{2}} ; \frac{M_{H}^{2}}{\mu_{F}^{2}} ; \frac{M_{H}^{2}}{m_{t}^{2}}\right) \exp \left\{\tilde{\mathcal{G}}_{H}\left(\alpha_{s}\left(\mu_{R}^{2}\right), \log N ; \frac{M_{H}^{2}}{\mu_{R}^{2}}, \frac{M_{H}^{2}}{\mu_{F}^{2}}\right)\right\}$

where we included top mass effects up to the NLL level explicitly as will be discussed in the following. The function $C_{g g}$ contains all constant terms that originate from the $\delta(1-z)$ terms of the explicit perturbative results and additional contributions emerging from the Mellin transformation. It develops a perturbative expansion in the strong coupling $\alpha_{s}$,

$$
C_{g g}\left(\alpha_{s}\left(\mu_{R}^{2}\right), \frac{M_{H}^{2}}{\mu_{R}^{2}} ; \frac{M_{H}^{2}}{\mu_{F}^{2}} ; \frac{M_{H}^{2}}{m_{t}^{2}}\right)=1+\sum_{n=1}^{\infty}\left(\frac{\alpha_{s}\left(\mu_{R}^{2}\right)}{\pi}\right)^{n} C_{g g}^{(n)}\left(\frac{M_{H}^{2}}{\mu_{R}^{2}}, \frac{M_{H}^{2}}{\mu_{F}^{2}} ; \frac{M_{H}^{2}}{m_{t}^{2}}\right) .
$$

The leading logarithmic terms in $N$ are contained in the exponential factor with the exponent

$$
\tilde{\mathcal{G}}_{H}\left(\alpha_{s}\left(\mu_{R}^{2}\right), \log N ; \frac{M_{H}^{2}}{\mu_{R}^{2}}, \frac{M_{H}^{2}}{\mu_{F}^{2}}\right)=\log N g_{H}^{(1)}(\lambda)+\left.\sum_{n=2}^{\infty} \alpha_{s}^{n-2}\left(\mu_{R}^{2}\right) g_{H}^{(n)}\left(\lambda, \frac{M_{H}^{2}}{\mu_{R}^{2}} ; \frac{M_{H}^{2}}{\mu_{F}^{2}}\right)\right|_{\lambda=b_{0} \alpha_{s}\left(\mu_{R}^{2}\right) \log N}
$$

where $b_{0}$ denotes the leading order term of the QCD beta function,

$$
b_{0}=\frac{33-2 N_{F}}{12 \pi}
$$

\footnotetext{
${ }^{2}$ Note that the soft and collinear limits of the mass-dependent functions $d_{i j}^{H}\left(z, \tau_{Q}\right)$ coincide with the heavy top limits presented in Eq. (5).
} 
where $N_{F}$ is the number of active flavors that we choose as $N_{F}=5$ in the following; i.e. the top quark has been decoupled from the strong coupling $\alpha_{s}$ and the PDFs. The term containing the function

$$
g_{H}^{(1)}(\lambda)=\frac{3}{\pi b_{0} \lambda}[2 \lambda+(1-2 \lambda) \log (1-2 \lambda)]
$$

resums the leading logarithms in $N$, while the successive terms with $g_{H}^{(i)}(i \geq 2)$ cover the subleading, subsubleading, etc. contributions. The explicit expressions for $g_{H}^{(2)}$ and $g_{H}^{(3)}$ can be found in Refs. $[18,47]$ and the one for $g_{H}^{(4)}$ in [48] where the four-loop cusp anomalous dimension has been approximated by employing Padé approximants. In the following we will use this approximate expression for $g_{H}^{(4)}$. The NLO coefficient of the function $C_{g q}$ is given by [18]

$$
\begin{aligned}
C_{g g}^{(1)} & =\delta G_{g g}^{(1)}+6\left(\gamma_{E}^{2}+\zeta_{2}\right)-6 \gamma_{E} \log \frac{M_{H}^{2}}{\mu_{F}^{2}} \\
\delta G_{g g}^{(1)} & =c_{H}\left(\tau_{t}\right)+6 \zeta_{2}+\frac{33-2 N_{F}}{6} \log \frac{\mu_{R}^{2}}{\mu_{F}^{2}}
\end{aligned}
$$

where we included the top quark mass dependence explicitly by using the function $c_{H}\left(\tau_{t}\right)$ [20]. The NNLO coefficient $C_{g g}^{(2)}$ in the limit of heavy top quarks can be found in Ref. [18]. The $\mathrm{N}^{3} \mathrm{LO}$ term can be extracted from the explicit results given in [21] and can be found in Appendix E of [49]. In Ref. [12] the inclusion of additional terms of purely collinear origin in the resummation has been discussed and then implemented in the results of $[15,18]$ by performing the replacement

$$
C_{g g}^{(1)} \rightarrow C_{g g}^{(1)}+6 \frac{\log N}{N} .
$$

This replacement reproduces all leading collinear logarithms of the form $\alpha_{s}^{n} \log ^{2 n-1} N / N$ up to all orders. However, the subleading logarithms of this type are not covered by this replacement as can be inferred from the explicit NNLO expansion of the resummed expression in Ref. [18]. We have observed that the subleading logarithms of the type $\gamma_{E} \log ^{2} N / N$ and $\log M_{H}^{2} / \mu_{F}^{2} \log ^{2} N / N$ can be reproduced by the extended replacement

$$
C_{g g}^{(1)} \rightarrow C_{g g}^{(1)}+6 \frac{\tilde{L}}{N}
$$

with the modified logarithm

$$
\tilde{L}=\log \frac{N e^{\gamma_{E}} \mu_{F}}{M_{H}}=\log N+\gamma_{E}-\frac{1}{2} \log \frac{M_{H}^{2}}{\mu_{F}^{2}} .
$$

This method can be extended to the next perturbative order. By performing the NNLO replacement

$$
C_{g g}^{(2)} \rightarrow C_{g g}^{(2)}+\left(48-N_{F}\right) \frac{\tilde{L}^{2}}{N}
$$

we reproduce the correct collinear logarithms $\log ^{i} N / N$ for $i=5,4$ at $\mathrm{N}^{3} \mathrm{LO}$,

$$
\begin{aligned}
G_{g g}^{(3)}= & 36 \log ^{6} N+170.679 \log ^{5} N \\
& +744.849 \log ^{4} N+1405.185 \log ^{3} N \\
& +2676.129 \log ^{2} N+1897.141 \log N+1783.692 \\
& +\frac{1}{N}\left\{108 \log ^{5} N+615.696 \log ^{4} N\right\}+\ldots
\end{aligned}
$$

where all numbers have been rounded at $\mathcal{O}\left(10^{-3}\right)$ and the additional contributions of the effective Lagrangian in the heavy top limit have not been taken into account as in Ref. [21]. These terms agree with the threshold expansion of the $\mathrm{N}^{3} \mathrm{LO}$ results of [21] and the logarithmic scale dependence terms up to the first two leading logarithms of $\mathcal{O}(1 / N)$. A further inclusion of subsubleading collinear logarithms requires a modified systematic expansion of the Mellin transform of the resummed kernel in $\log \tilde{N}=$ $\log \left(N e^{\gamma_{E}}\right)$ instead of $\log N$ and powers of $N^{-1}$ which is beyond the scope of this work. It should be noted that the extension of the resummation by the collinear terms of Eqs. (19)-(21) is still an unproven conjecture that, however, is corroborated by the explicit reproduction of the subleading logarithmic structure up to $\mathrm{N}^{3} \mathrm{LO}$. Our approach can be compared with the equivalent approach of Ref. [50] that tries to construct a second exponential resummation for the $\mathcal{O}(1 / N)$ logarithms to be added to the leading soft + virtual exponential. However, Ref. [50] does not apply their conjecture to the Higgs boson case so that a direct comparison of the methods is not possible presently. On the other hand we have found numerical agreement with the results of Ref. [25] at the percent level. Minor differences are expected due to the slightly different resummation methods applied.

The numerical impact of the subleading collinear logarithms and top mass effects in the resummed expression ranges in the sub-per-mille range for the SM Higgs mass. The inclusion of top mass effects in the resummation can reach the percent level beyond NLO for large Higgs masses. An alternative implementation of resummation effects includes the full coefficient $C_{g g}$ of Eq. (12) in the resummed exponential with a careful expansion of the exponent up to all terms of $\mathcal{O}(1 / N)$ included in the analysis along the lines of Ref. [12]. We have checked that this modification has an impact in the per-mille range on the final results for the inclusive SM Higgs cross sections.

\section{B. Mass effects and matching}

Using the resummed expression of the gluon-fusion cross section in Mellin space we subtract the corresponding 
fixed-order Mellin-space result up to NNLO in order to obtain the net effect of resummation beyond NNLO. This residual contribution has then been added to the NNLO result that has been obtained by including the full NNLO result in the limit of heavy top quarks for the pure top quark contributions and adding top mass effects and bottom (charm) contributions at NLO strictly. Furthermore we include only the top quark mass effects in the resummed cross section at the NLL level (convolved with NLO $\alpha_{s}$ and PDFs) and treat the bottom- and charm-induced parts at fixed NLO. Since the virtual coefficient of the bottom contributions behaves in the limit $M_{H}^{2} \gg m_{b}^{2}$ as [9] $\left(C_{A}=3, C_{F}=4 / 3\right)$

$$
c_{H}\left(\tau_{b}\right) \rightarrow \frac{C_{A}-C_{F}}{12} \log ^{2} \frac{M_{H}^{2}}{m_{b}^{2}}-C_{F} \log \frac{M_{H}^{2}}{m_{b}^{2}}
$$

if the bottom mass is renormalized on shell, it contains large logarithms that are not resummed. The resummation of the
Abelian part proportional to $C_{F}$ has been performed in Ref. [51] up to the subleading logarithmic level. These logarithms are related to the Sudakov form factor at the virtual $H b \bar{b}$ vertex that generates these large logarithmic contributions for far off-shell bottom quarks inside the corresponding loop contributions in the Abelian case. The resummation of the non-Abelian part proportional to the Casimir factor $C_{A}$ has not been considered so far. This type of logarithmic contribution emerges from a different origin than the soft and collinear gluon effects discussed so far and is the main source of the very different size of QCD corrections to the bottom-loop contributions [9-11]. In order to obtain a reliable result for the bottom contributions a resummation of these types of logarithms is mandatory so that we do not include these contributions in our soft and collinear gluon resummation but treat them at fixed NLO.

The complete cross section including soft/collinear gluon resummation effects can then be cast into the generic form

$$
\begin{aligned}
\sigma\left(s, M_{H}^{2}\right)= & \sigma_{t t}^{0} \sum_{i j} \int_{C-i \infty}^{C+i \infty} \frac{d N}{2 \pi i}\left(\frac{M_{H}^{2}}{s}\right)^{-N+1} \tilde{f}_{g}\left(N, \mu_{F}^{2}\right) \tilde{f}_{g}\left(N, \mu_{F}^{2}\right) \\
& \times\left\{\tilde{G}_{g g}^{\text {(res) }}\left(N ; \alpha_{s}\left(\mu_{R}^{2}\right), \frac{M_{H}^{2}}{\mu_{R}^{2}} ; \frac{M_{H}^{2}}{\mu_{F}^{2}} ; 0\right)-\left[\tilde{G}_{g g}^{\text {(res) }}\left(N ; \alpha_{s}\left(\mu_{R}^{2}\right), \frac{M_{H}^{2}}{\mu_{R}^{2}} ; \frac{M_{H}^{2}}{\mu_{F}^{2}} ; 0\right)\right]_{(\mathrm{NNLO})}\right\} \\
& +\sigma_{t t}^{0} \sum_{i j} \int_{C-i \infty}^{C+i \infty} \frac{d N}{2 \pi i}\left(\frac{M_{H}^{2}}{s}\right)^{-N+1} \tilde{f}_{g}\left(N, \mu_{F}^{2}\right) \tilde{f}_{g}\left(N, \mu_{F}^{2}\right) \\
& \times\left\{\tilde{G}_{g g, \mathrm{NLL}}^{\text {(res })}\left(N ; \alpha_{s}\left(\mu_{R}^{2}\right), \frac{M_{H}^{2}}{\mu_{R}^{2}} ; \frac{M_{H}^{2}}{\mu_{F}^{2}} ; \frac{M_{H}^{2}}{m_{t}^{2}}\right)-\tilde{G}_{g g, \mathrm{NLL}}^{\text {(res })}\left(N ; \alpha_{s}\left(\mu_{R}^{2}\right), \frac{M_{H}^{2}}{\mu_{R}^{2}} ; \frac{M_{H}^{2}}{\mu_{F}^{2}} ; 0\right)\right. \\
& \left.\left.-\left[\tilde{G}_{g g, N L L}^{(\mathrm{res})}\left(N ; \alpha_{s}\left(\mu_{R}^{2}\right), \frac{M_{H}^{2}}{\mu_{R}^{2}} ; \frac{M_{H}^{2}}{\mu_{F}^{2}} ; \frac{M_{H}^{2}}{m_{t}^{2}}\right)-\tilde{G}_{g g, \mathrm{NLL}}^{(\mathrm{res})}\left(N ; \alpha_{s}\left(\mu_{R}^{2}\right), \frac{M_{H}^{2}}{\mu_{R}^{2}} ; \frac{M_{H}^{2}}{\mu_{F}^{2}} ; 0\right)\right]\right]_{(\mathrm{NLO})}\right\} \\
& +\sigma_{t+b+c}^{\mathrm{NNLO}}\left(s, M_{H}^{2}\right)
\end{aligned}
$$

with $\sigma_{t t}^{0}$ denoting the LO cross section factor $\sigma_{0}$ of Eq. (1) including only the top quark contribution. The index "(NNLO $) "$ in the second line indicates the fixed-order expansion of the resummed coefficient function $\tilde{G}_{g g}^{(\text {res })}$ in Mellin space up to NNLO while the index " $(N L O)$ " denotes the perturbative expansion of the NLL resummed coefficient function $\tilde{G}_{g g, \mathrm{NLL}}^{\text {(res) }}$ in Mellin space up to NLO. The first integral has been convolved with $\mathrm{N}^{3} \mathrm{LO} \alpha_{s}$ and NNLO PDFs according to the discussion about the non-necessity of $\mathrm{N}^{3}$ LO PDFs of Ref. [52] and of resummed PDFs of Ref. [53] for the SM Higgs mass, while the second integral has been evaluated with NLO $\alpha_{s}$ and PDFs consistently. The fixedorder NNLO cross section of the last term has been derived as

$$
\begin{aligned}
\sigma_{t+b+c}^{\mathrm{NNLO}}\left(s, M_{H}^{2}\right)= & \sigma_{\infty}^{\mathrm{NNLO}}\left(s, M_{H}^{2}\right)+\sigma_{t+b+c}^{\mathrm{NLO}}\left(s, M_{H}^{2}\right) \\
& -\sigma_{\infty}^{\mathrm{NLO}}\left(s, M_{H}^{2}\right)
\end{aligned}
$$

where the individual parts are defined as

$$
\begin{aligned}
\sigma_{\infty}^{\mathrm{NNLO}}\left(s, M_{H}^{2}\right) & =\sigma_{t t}^{\mathrm{LO}} K_{\infty}^{\mathrm{NNLO}} \\
\sigma_{\infty}^{\mathrm{NLO}}\left(s, M_{H}^{2}\right) & =\sigma_{t t}^{\mathrm{LO}} K_{\infty}^{\mathrm{NLO}} \\
\sigma_{t+b+c}^{\mathrm{NLO}}\left(s, M_{H}^{2}\right) & =\sigma_{t+b+c}^{\mathrm{LO}} K_{t+b+c}^{\mathrm{NLO}}
\end{aligned}
$$

where $\sigma_{t t}^{\mathrm{LO}}$ denotes the full LO cross section including only top loops, $\sigma_{t+b+c}^{\mathrm{LO}}$ the LO cross section including top and bottom/charm loops, $K_{\infty}^{(N) N L O}$ the (N)NLO K-factors obtained in the limit of heavy top quarks and $K_{t+b+c}^{\mathrm{NLO}}$ the full NLO K-factor including top and bottom/charm loops. The NNLO parts have been derived with $\mathrm{N}^{3} \mathrm{LO} \alpha_{s}$ and NNLO PDFs and the NLO terms with NLO $\alpha_{s}$ and PDFs consistently as implemented in the programs HIGLU [54] and SusHi [55]. This implementation guarantees that top mass effects 
are treated at the NLL level and bottom/charm contributions at fixed NLO respectively.

\section{Mellin inversion}

As a final step we have to perform the Mellin inversion of the resummed and properly matched result in Mellin space. According to Eq. (24) this requires the inclusion of the Mellin moments of the gluon densities $\tilde{f}_{q}\left(N, \mu_{F}^{2}\right)$ in the integrand for the contour integration. This can be treated in different ways. References $[18,20]$ used a fit to the parton densities in Bjorken- $x$ space for a fixed factorization scale and used this fit to determine the Mellin moments analytically in terms of a set of fitted coefficients. We did not proceed along these lines. A second option will be to use the evolution program PEGASUs [56] in Mellin space that just requires the implementation of the input densities in terms of a predefined functional form. The latter does not, however, coincide with the input densities of all available global PDF fits. We have performed cross checks with PEGASUS for the MSTW08 PDFs [57] at LO, NLO and NNLO. ${ }^{3}$ The method that we adopted in our numerical analysis is to implement the Mellin moments of the
Bjorken- $x$ PDFs by a numerical integration. The fact that the $z$-integral of the fixed-order integral is extended to an infinite upper bound after integration [58] due to the presence of the Landau singularity at $N_{L}=$ $\exp \left(1 /\left[2 b_{0} \alpha_{s}\left(\mu_{R}^{2}\right)\right]\right)$ implies that the resummed kernel $\tilde{G}_{g g}^{\text {(res) }}$ does not vanish for $z>1$ but drops down very fast for larger values of $z$. In order to increase the numerical stability of the Mellin inversion we have included four additional powers $1 /(N-1)^{4}$ in the resummed kernels so that the large $N$ contributions for $z>1$ are sufficiently suppressed. By means of partial integrations this can be translated to the convolution over derivatives of the gluon PDFs [59],

$$
\begin{aligned}
& \tilde{\sigma}\left(N-1, M_{H}^{2}\right) \\
& \quad=\sigma_{0} \tilde{\mathcal{F}}_{g}\left(N, \mu_{F}^{2}\right) \tilde{\mathcal{F}}_{g}\left(N, \mu_{F}^{2}\right) \tilde{\mathcal{G}}_{g g}^{(\text {res })}\left(N ; \alpha_{s}\left(\mu_{R}^{2}\right), \frac{M_{H}^{2}}{\mu_{R}^{2}} ; \frac{M_{H}^{2}}{\mu_{F}^{2}}\right)
\end{aligned}
$$

with

$$
\begin{aligned}
\tilde{\mathcal{G}}_{g g}^{\text {(res })}\left(N ; \alpha_{s}\left(\mu_{R}^{2}\right), \frac{M_{H}^{2}}{\mu_{R}^{2}} ; \frac{M_{H}^{2}}{\mu_{F}^{2}}\right) & =\tilde{G}_{g g}^{(\mathrm{res})}\left(N ; \alpha_{s}\left(\mu_{R}^{2}\right), \frac{M_{H}^{2}}{\mu_{R}^{2}} ; \frac{M_{H}^{2}}{\mu_{F}^{2}}\right) /(N-1)^{4} \\
\tilde{\mathcal{F}}_{g}\left(N, \mu_{F}^{2}\right) & =(N-1)^{2} \tilde{f}_{g}\left(N, \mu_{F}^{2}\right)=\int_{0}^{1} d x x^{N-1} \frac{d}{d x}\left\{x \frac{d}{d x}\left[x f_{g}\left(x, \mu_{F}^{2}\right)\right]\right\} .
\end{aligned}
$$

The second derivative has been implemented by an equidistant three-point method

$$
f^{\prime \prime}(x)=\frac{f(x+h)-2 f(x)+f(x-h)}{h^{2}}+\mathcal{O}\left(h^{2}\right) .
$$

Using this method we can implement the original PDF fits in Bjorken- $x$ space and obtain sufficient numerical stability if the contour is chosen according to the parametrization [59]

$$
N=C+x e^{ \pm i \phi}
$$

with the $+(-)$ sign applied to the upper (lower) contour with respect to the real axis and the integration proceeds over the variable $x$. The offset parameter has been chosen as $C=2.5$

\footnotetext{
${ }^{3}$ Differences between the original MSTW08 evolution and the results of PEGASUS at large Bjorken- $x$ have been clarified after including an updated set of MSTW08 PDFs [49]. The impact on the gluon-fusion cross section is small, i.e. at the per-mille level both for small and large Higgs masses, and has been neglected in this analysis.

${ }^{4}$ Note that the translation to the second derivative appearing in the Mellin integral assumes that the gluon density and its first derivative vanish for $x=1$.
}

and the angle $\phi$ as $3 \pi / 4$ for $z<1$ and $\pi / 4$ for $z>1$. This choice ensures that all relevant singularities in Mellin space are located to the left of the integration contour, but the Landau singularity is to the right; i.e. the latter is excluded from the contour integral according to the minimal prescription method of Ref. [58]. We have checked the independence of our results of variations of the two parameters $C, \phi$ around the chosen values within the valid ranges, i.e. keeping $C$ in the range $2<C<N_{L}$ and the signs of the angle $\phi$ and its hemispheres with respect to $\pi / 2$.

\section{MSSM HIGGS BOSON PRODUCTION}

In the MSSM all three neutral Higgs bosons are produced via gluon fusion $g g \rightarrow h, H, A$. In the following we will neglect the top-squark and sbottom loops in MSSM scalar Higgs boson production and will focus on the topand bottom-induced contributions that are modified in the scalar case due to the additional mixing factors of the top and bottom Yukawa couplings compared to the SM Higgs case as given in Table I. This approximation works for heavy top squarks and sbottoms. Pseudoscalar Higgs boson production, however, requires a transformation of the SM Higgs results to the production of a $\mathcal{C P}$-odd Higgs particle. The resummed exponential of Eq. (12) is universal and thus 
unchanged in the pseudoscalar case (if $M_{H}$ is replaced by the pseudoscalar Higgs mass $M_{A}$ ) so that only the coefficient $C_{g g}$ is different from scalar Higgs boson production. The difference of this coefficient between the pseudoscalar and scalar Higgs cases is given by ${ }^{5}$

$$
\begin{aligned}
\Delta C_{g g}^{(1)}= & C_{g g, A}^{(1)}-C_{g g, H}^{(1)}=c_{A}\left(\tau_{t}\right)-c_{H}\left(\tau_{t}\right) \\
\Delta C_{g g}^{(2)}= & C_{g g, A}^{(2)}-C_{g g, H}^{(2)} \\
= & \frac{1939}{144}+3 \gamma_{E}^{2}+6 \zeta_{2}-\frac{21}{16} N_{F}-\left(\frac{19}{8}-\frac{N_{F}}{3}\right) \log \frac{M_{A}^{2}}{m_{t}^{2}} \\
& +\left(\frac{33-2 N_{F}}{12}-3 \gamma_{E}\right) \log \frac{M_{A}^{2}}{\mu_{F}^{2}}-\frac{33-2 N_{F}}{8} \log \frac{M_{A}^{2}}{\mu_{R}^{2}}
\end{aligned}
$$

where at NLO we again include the full top mass dependence in this coefficient along the lines of Ref. [20]. In the limit of heavy top quarks the difference of the virtual corrections at NLO approaches

$$
\Delta C_{g g}^{(1)}=C_{g g, A}^{(1)}-C_{g g, H}^{(1)} \rightarrow \frac{1}{2} .
$$

Since the gluon-fusion production cross section for pseudoscalar Higgs bosons is only known up to NNLO we reduce our resummation to the NNLL level; i.e. we do not include the function $g_{H}^{(4)}$ in the resummed exponent of Eq. (14). The inclusion of purely collinear logarithms proceeds along the same lines as for the SM Higgs case by implementing the replacements

$$
\begin{aligned}
C_{g g, A}^{(1)} & \rightarrow C_{g g, A}^{(1)}+6 \frac{\tilde{L}}{N} \\
C_{g g, A}^{(2)} & \rightarrow C_{g g, A}^{(2)}+\left(48-N_{F}\right) \frac{\tilde{L}^{2}}{N}
\end{aligned}
$$

with the extended logarithm

$$
\tilde{L}=\log \frac{N e^{\gamma_{E}} \mu_{F}}{M_{H}}=\log N+\gamma_{E}-\frac{1}{2} \log \frac{M_{A}^{2}}{\mu_{F}^{2}} .
$$

These replacements resum the corresponding collinear logarithms at leading and subleading level accordingly.

\section{RESULTS}

We analyze Higgs boson production via gluon fusion at the LHC for a c.m. energy of $\sqrt{s}=13 \mathrm{TeV}$ for the SM Higgs boson and the neutral Higgs bosons of the MSSM at the $\mathrm{N}^{3} \mathrm{LL}$ level for the top-induced contributions of the

\footnotetext{
${ }^{5}$ Note that the NNLO expression differs from Ref. [38]. The (numerically minor) discrepancies have been clarified with the authors.
}

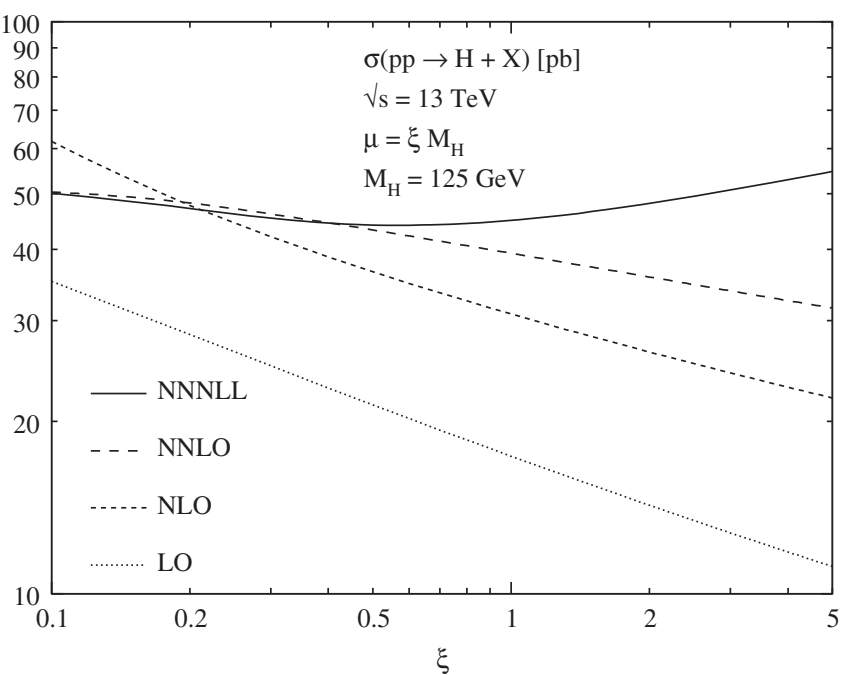

FIG. 1. Scale dependence of the SM Higgs production cross sections via gluon fusion for a Higgs mass $M_{H}=125 \mathrm{GeV}$ and c.m. energy $\sqrt{s}=13 \mathrm{TeV}$ using MSTW08 PDFs. The renormalization and factorization scales are identified, i.e. $\mu=\mu_{R}=\mu_{F}$.

scalar Higgs cases and at the NNLL level for the pseudoscalar case. As parton density functions (PDFs) we will primarily use the MSTW08 sets [57] with the strong coupling normalized to $\alpha_{s}\left(M_{Z}^{2}\right)=0.12018$ at NLO and to $\alpha_{s}\left(M_{Z}^{2}\right)=0.11707(\mathrm{~N}) \mathrm{NNLO}$. The quark pole masses have been chosen as $m_{t}=172.5 \mathrm{GeV}, m_{b}=4.75 \mathrm{GeV}$ and $m_{c}=1.40 \mathrm{GeV}$ accordingly. For the determination of the $\mathrm{PDF}+\alpha_{s}$ uncertainties we have used the envelope method [60] with CT10 [61] and NNPDF2.3 [62] PDF sets. For CT10 the strong coupling has been normalized consistently to $\alpha_{s}=0.118$ and for NNPDF2.3 to $\alpha_{s}=0.119$, respectively. The uncertainty in the strong coupling constant has been adopted as \pm 0.002 around the corresponding central values of the used PDFs. ${ }^{6}$

\section{A. Standard Model}

For the SM Higgs boson we perform the numerical analysis for Higgs mass values around the measured Higgs mass of about $125 \mathrm{GeV}$. For the uncertainties we consider the scale dependence and the $\mathrm{PDF}+\alpha_{s}$ uncertainties. The scale dependence at LO, NLO, NNLO and $\mathrm{N}^{3} \mathrm{LL}$ is shown in Fig. 1 as a function of the identified renormalization and factorization scales in units of the Higgs mass $M_{H}$. We

\footnotetext{
${ }^{6}$ If other PDF sets as ABM12 [63] or HERAPDF1.5 [64] are included in this envelope the $\mathrm{PDF}+\alpha_{s}$ uncertainties will increase considerably with a major part originating from sizable differences in the $\alpha_{s}$ fits at NNLO and different data sets included in the global fits. Moreover, the proper treatment of higher-twist effects in the global fits is an open aspect in this context. However, we have adopted the scheme used within the HXSWG, since an extended study of this particular issue is beyond the scope of our paper.
} 
TABLE II. N $\mathrm{N}^{3}$ LL Higgs boson production cross sections via gluon fusion for different values of the renormalization and factorization scales $\mu_{R}, \mu_{F}$ without electroweak corrections for a Higgs mass $M_{H}=125 \mathrm{GeV}$ and c.m. energy $\sqrt{s}=13 \mathrm{TeV}$.

\begin{tabular}{lccc}
\hline \hline \multicolumn{4}{c}{$\mu_{R} / M_{H}$} \\
\hline$\mu_{F} / M_{H}$ & $1 / 4$ & $1 / 2$ & 1 \\
\hline $1 / 4$ & $46.08 \mathrm{pb}$ & $42.92 \mathrm{pb}$ & $39.35 \mathrm{pb}$ \\
$1 / 2$ & $45.42 \mathrm{pb}$ & $44.13 \mathrm{pb}$ & $41.81 \mathrm{pb}$ \\
1 & $44.98 \mathrm{pb}$ & $45.81 \mathrm{pb}$ & $44.94 \mathrm{pb}$ \\
\hline \hline
\end{tabular}

observe a significant decrease of the scale dependence with increasing perturbative order signaling a rather mild scale dependence at $\mathrm{N}^{3} \mathrm{LL}$. However, this is caused by a significant cancellation between the renormalization and factorization scale dependences that develop opposite slopes [18]. For a consistent estimate of the theoretical uncertainties both scales have to be varied independently. The results of varying both scales by a factor of two up and down around our central scale choice $\mu_{0}=M_{H} / 2$ are shown in Table II without electroweak corrections. The numbers develop a much larger variation within the complete interval of independent variations so that identifying the renormalization and factorization scales leads to a significant underestimate of the related uncertainty. Taking the minimal and maximal values of Table II excluding the values for $\mu_{F} / \mu_{R}>2$ and $\mu_{F} / \mu_{R}<\frac{1}{2}$ we derive a total scale dependence of $[+4.4 \%,-5.3 \%]$, while for identified scales we obtain the optimistic estimate of $[+4.4 \%,-0.1 \%]$. Thus for a sophisticated determination of the theoretical uncertainties due to the scale dependence both scales have to be varied independently.

Including electroweak corrections [27] we present our predictions of the central cross sections for five different Higgs mass values in Table III where we choose the MSTW08 PDFs [57] and add the corresponding PDF $+\alpha_{s}$ uncertainties. The total uncertainty is obtained by adding the scale and $\mathrm{PDF}+\alpha_{s}$ uncertainties linearly. In this way the total uncertainties amount to $\mathcal{O}(10 \%)$. Additional

TABLE III. N $\mathrm{N}^{3}$ LL Higgs boson production cross sections via gluon fusion for different values of the Higgs mass including the individual uncertainties due to the renormalization and factorization scale dependence and $\mathrm{PDF}+\alpha_{s}$ uncertainties including electroweak corrections using MSTW08 PDFs for a c.m. energy $\sqrt{s}=13 \mathrm{TeV}$.

\begin{tabular}{lcccc}
\hline \hline$M_{H}(\mathrm{GeV})$ & $\sigma(p p \rightarrow H+X)(\mathrm{pb})$ & Scale & $\mathrm{PDF}+\alpha_{s}$ & Total \\
\hline 124 & 47.05 & $+4.5 \%$ & $+3.7 \%$ & $+8.2 \%$ \\
124.5 & 46.72 & $-5.2 \%$ & $-4.0 \%$ & $-9.2 \%$ \\
125 & 46.40 & $-5.4 \%$ & $+3.7 \%$ & $+8.1 \%$ \\
125.5 & 46.06 & $-4.4 \%$ & $-4.0 \%$ & $-9.3 \%$ \\
126 & 45.74 & $-5.3 \%$ & $-4.0 \%$ & $-9.1 \%$ \\
& & $-5.4 \%$ & $+3.7 \%$ & $+8.1 \%$ \\
& & $+4.4 \%$ & $-4.0 \%$ & $-9.2 \%$ \\
\hline \hline
\end{tabular}

TABLE IV. N $\mathrm{N}^{3}$ LL Higgs boson production cross sections via gluon fusion for different values of the Higgs mass including the individual and total uncertainties due to the renormalization and factorization scale dependence and $\mathrm{PDF}+\alpha_{s}$ uncertainties including electroweak corrections using the envelope of MSTW08 [57], CT10 [61] and NNPDF2.3 [62] PDFs for a c.m. energy $\sqrt{s}=13 \mathrm{TeV}$.

\begin{tabular}{lcccc}
\hline$M_{H}(\mathrm{GeV})$ & $\sigma(p p \rightarrow H+X)(\mathrm{pb})$ & Scale & PDF $+\alpha_{s}$ & Total \\
\hline 124 & 48.37 & $+4.5 \%$ & $\pm 8.1 \%$ & $+12.6 \%$ \\
124.5 & 48.00 & $-5.2 \%$ & $-13.3 \%$ \\
125 & 47.63 & $-5.4 \%$ & $\pm 8.1 \%$ & $+12.5 \%$ \\
125.5 & 47.28 & $-4.4 \%$ & $\pm 8.1 \%$ & $-13 \%$ \\
126 & $46.4 \%$ \\
12.94 & $-5.4 \%$ & $\pm 8.2 \%$ & $-13 \%$ \\
& $4.42 \%$ \\
\hline \hline
\end{tabular}

uncertainties due to parametric uncertainties in the top and bottom/charm quark masses and missing quark mass effects beyond NLO are small, i.e. in the percent range and thus negligible. In Table IV we present our final predictions of the gluon fusion cross section for five different Higgs masses with the central values and $\mathrm{PDF}+\alpha_{s}$ obtained by the envelope method [60] involving MSTW08 [57], CT10 [61] and NNPDF2.3 [62] PDFs. This results in a slight increase of the total uncertainties to the level of $\mathcal{O}(15 \%)$. This situation will improve with the inclusion of more recent PDFs including LHC data in their global fits.

Finally, we compare our results with the $\mathrm{N}^{3} \mathrm{LO}$ predictions of Ref. [22] in Table V. This table shows the numbers of Ref. [22] in the first column which have been obtained in the limit of heavy top quarks for the QCD corrected cross sections at all perturbative orders and neglecting the bottom and charm loops. The renormalization and factorization scales have been identified for the derivation of the scale dependence given in percent attached to each number. The second column labeled " $\mathrm{SVC}_{\infty}$ " depicts our prediction in the heavy top limit by using the approximate soft + virtual + collinear expansion of our resummed kernel for the $\mathrm{N}^{3} \mathrm{LO}$ piece added to the full NNLO result in the same limit. These approximate fixed-order results agree with the explicit numbers of Ref. [22] within $0.2 \%$ for $\mu_{R}=\mu_{F}=$ $M_{H} / 2$ and within $1.5 \%$ for $\mu_{R}=\mu_{F}=M_{H}$ and develop a similar scale dependence. Our corresponding result at $\mathrm{N}^{3} \mathrm{LL}$ is shown in the third column labeled " $\mathrm{N}^{3} \mathrm{LL}_{\infty}$ " in the limit of heavy top quarks and omitting the bottom and charm contributions. Our scale dependence for identified scales is of similar size as the one of the $\mathrm{N}^{3} \mathrm{LO}$ results. However, this scale dependence does not constitute a reliable estimate of the theoretical uncertainties as discussed before. The comparison of the second and third column shows the effect of resummation beyond $\mathrm{N}^{3} \mathrm{LO}$. It is clearly visible that the resummation effects range at the per-mille level for the scale choice $\mu_{R}=\mu_{F}=M_{H} / 2$, while resummation provides a $2 \%$ contribution beyond $\mathrm{N}^{3} \mathrm{LO}$ for the scale choice $\mu_{R}=\mu_{F}=M_{H}$. The latter effect 
TABLE V. Higgs boson production cross sections via gluon fusion for different values of the identified renormalization and factorization scales $\mu=\mu_{R}=\mu_{F}$ with and without quark mass effects and electroweak corrections in comparison to the $\mathrm{N}^{3} \mathrm{LO}$ results of Ref. [22] using MSTW08 PDFs for a c.m. energy $\sqrt{s}=13 \mathrm{TeV}$. The percentage errors provide the relative scale dependence for identified renormalization and factorization scales $\mu=\mu_{R}=\mu_{F}$ varied by a factor of two up and down around the central scale choice. The second and third columns of cross sections in the heavy top limit at all perturbative orders, labeled "SVC ${ }_{\infty}$ " and " $\mathrm{N}^{3} \mathrm{LL}_{\infty}$," exclude bottom/charm contributions.

\begin{tabular}{lccccr}
\hline \hline$\mu / M_{H}$ & Ref. [22] & $\mathrm{SVC}_{\infty}$ & $\mathrm{N}^{3} \mathrm{LL}_{\infty}$ & Massive N ${ }^{3} \mathrm{LL}$ & With electroweak corrections \\
\hline $1 / 2$ & $44.31_{-2.6 \%}^{+0.3 \%} \mathrm{pb}$ & $44.22_{-1.0 \%}^{+4.2 \%} \mathrm{pb}$ & $44.15_{-0.1 \%}^{+4.6 \%} \mathrm{pb}$ & $44.13_{-0.1 \%}^{+4.4 \%} \mathrm{pb}$ & $46.40_{-0.1 \%}^{+4.4 \%} \mathrm{pb}$ \\
1 & $43.14_{-4.5 \%}^{+2.7 \%} \mathrm{pb}$ & $43.77_{-0.0 \%}^{+1.0 \%} \mathrm{pb}$ & $44.72_{-1.3 \%}^{+6.3 \%} \mathrm{pb}$ & $44.94_{-1.8 \%}^{+6.6 \%} \mathrm{pb}$ & $47.25_{-1.8 \%}^{+6.6 \%} \mathrm{pb}$ \\
\hline \hline
\end{tabular}

is of the order of the uncertainties related to the scale dependence. The fourth column presents our full $\mathrm{N}^{3} \mathrm{LL}$ results including top mass effects up to NLL and bottom/ charm loops up to NLO. The value for our central scale choice $\mu_{R}=\mu_{F}=M_{H} / 2$ agrees with the number of Ref. [22] within $0.3 \%$ by accident. The last column shows our numbers including the electroweak corrections of Ref. [27]. Our final results deviate by a few percent from the numbers presented in [24,25], since the first analysis works at approximate fixed $\mathrm{N}^{3} \mathrm{LO}$ and includes neither top mass effects beyond LO nor bottom/charm loops and in both analyses electroweak corrections have not been taken into account. Moreover, small differences can arise due to different implementations of the PDFs in the resummation framework in comparison with the second analysis [25].

\section{B. Minimal supersymmetric extension}

For neutral MSSM Higgs boson production via gluon fusion we have adopted the $m_{h}^{\bmod +}$ scenario of Ref. [65] which is defined as

$$
\begin{aligned}
M_{\mathrm{SUSY}} & =1 \mathrm{TeV}, \quad \mu=M_{2}=200 \mathrm{GeV}, \\
X_{t} & =1.6 M_{\mathrm{SUSY}}, \quad m_{\tilde{g}}=1.5 \mathrm{TeV}, \\
A_{b} & =A_{\tau}=A_{t}
\end{aligned}
$$

where $M_{\text {SUSY }}$ denotes the SUSY-breaking sfermion mass scale of the third generation, $\mu$ the Higgsino mass parameter, $M_{2}$ the wino mass parameter, $X_{t}=A_{t}-\mu \operatorname{tg} \beta$ the topsquark mixing parameter, $m_{\tilde{g}}$ the gluino mass and $A_{t, b, \tau}$ the trilinear SUSY-breaking couplings of the sfermions and Higgs fields. We use the renormalization-group-improved two-loop expressions for the Higgs masses and couplings of Ref. [66] which yield predictions for the Higgs boson masses that agree with the diagrammatic calculations of Ref. [30] within 3\%-4\% in general. Thus the leading oneand two-loop corrections have been included in the Higgs masses and the effective mixing angle $\alpha$.

In Fig. 2 we show the scalar and pseudoscalar production cross sections as functions of the corresponding Higgs masses at LO, NLO, NNLO and (N)NNLL for the (scalar) pseudoscalar MSSM Higgs bosons. Squark loops and genuine SUSY-QCD corrections have been neglected in this work. They can be added in a form factorized from the resummation effects which is left for future work. The resummation effects beyond NNLO amount to about 5\% for $\operatorname{tg} \beta=3$, where the top loop contributions are dominant, while for large values of $\operatorname{tg} \beta=30$ the effects are small,
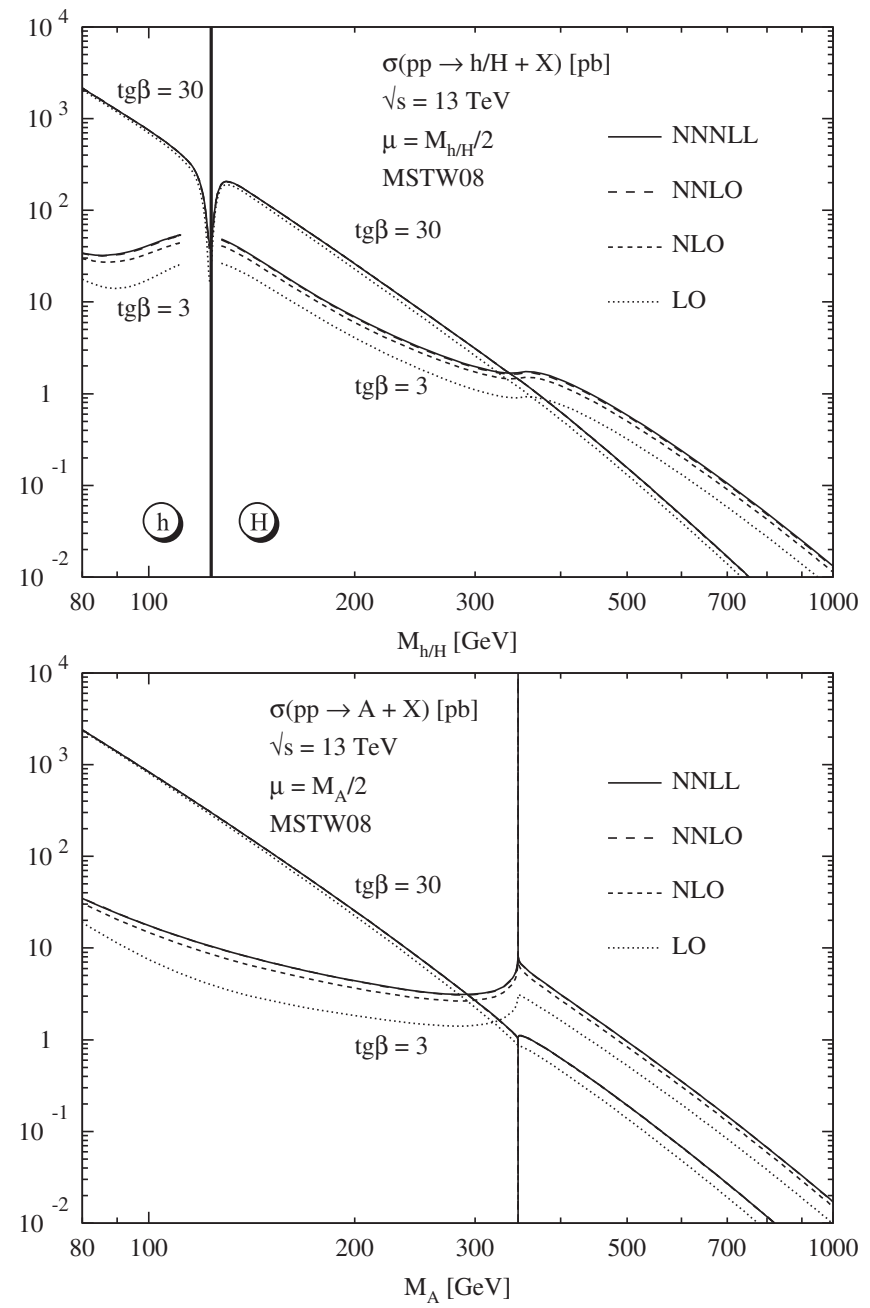

FIG. 2. MSSM Higgs production cross sections via gluon fusion at the LHC for a c.m. energy $\sqrt{s}=13 \mathrm{TeV}$ and two values of $\operatorname{tg} \beta=3,30$ using MSTW08 PDFs. For the MSSM the $m_{h}^{\text {mod }+}$ scenario [65] has been adopted and squark loops as well as genuine SUSY-QCD corrections have been neglected. 

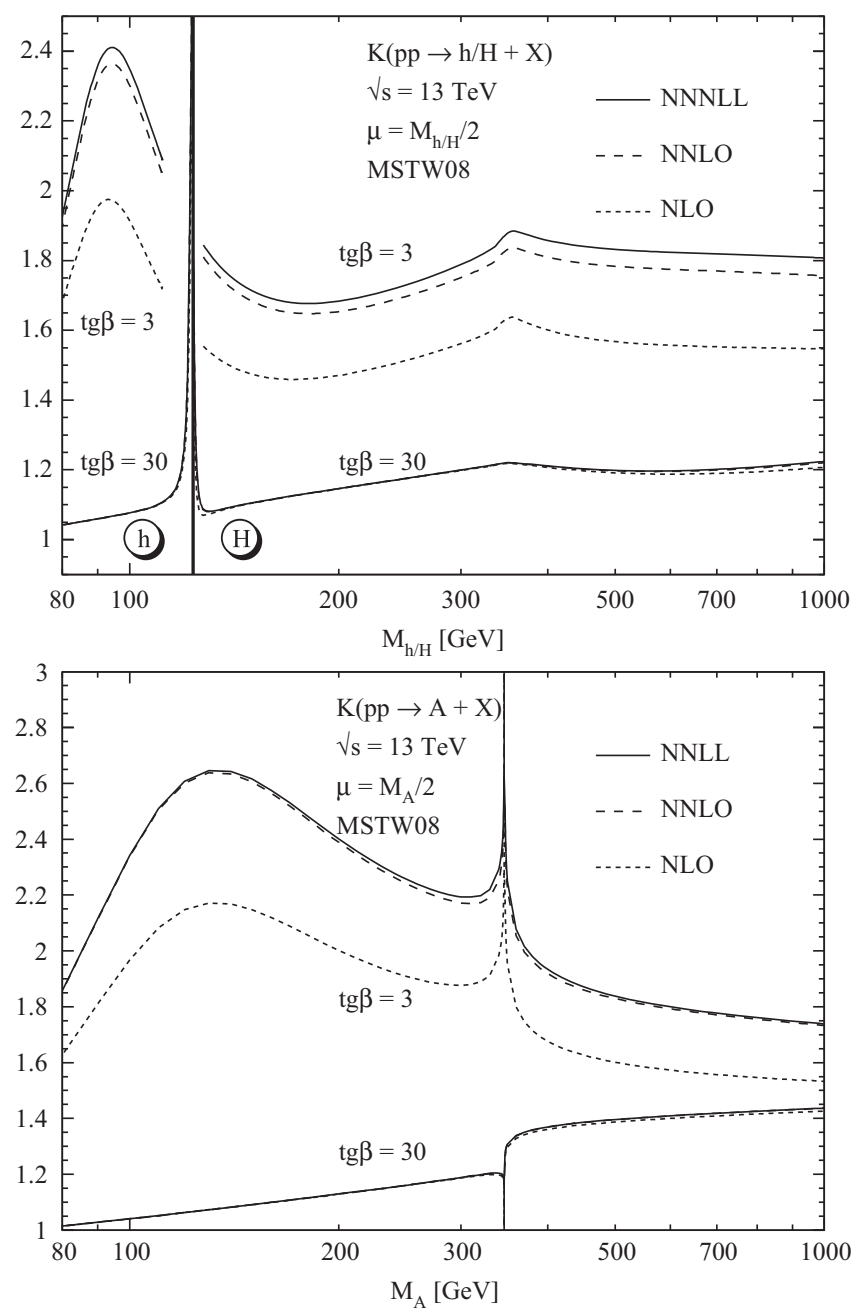

FIG. 3. K-factors of the MSSM Higgs production cross sections via gluon fusion at the LHC for a c.m. energy $\sqrt{s}=13 \mathrm{TeV}$ and two values of $\operatorname{tg} \beta=3,30$ using MSTW08 PDFs. For the MSSM the $m_{h}^{\bmod +}$ scenario [65] has been adopted and squark loops as well as genuine SUSY-QCD corrections have been neglected.

since the bottom loops dominate so that the accuracy of the cross section is of fixed NLO according to our setup as discussed in Sec. II B. This can be inferred more clearly from Fig. 3 which shows the corresponding K-factors for scalar and pseudoscalar Higgs boson production via gluon fusion. The K-factors are defined as the ratios of the NLO, NNLO and (N)NNLL cross sections to the LO prediction where each order is consistently evaluated with the corresponding PDF and $\alpha_{s}$ choices. For large values of $\operatorname{tg} \beta=30$ it is obvious from this figure that there is only a tiny effect beyond NLO as expected due to the dominance of the bottom loops that are purely treated at fixed NLO. The bumps and spikes for $M_{H / A} \sim 2 m_{t}$ are related to the $t \bar{t}$ threshold that generates a Coulomb singularity for the pseudoscalar case already at NLO [9,36]. The latter is regularized by taking into account the finite width of the virtual top quarks which, however, is beyond the scope of our work. The results indicate that the QCD uncertainties reduce to the level of $\sim 10 \%$ after including the resummation effects in regions of top-loop dominance. The dominant uncertainties in these regions within the MSSM will arise from genuine SUSY effects.

\section{CONCLUSIONS}

In this work we have performed an analysis of soft and collinear gluon resummation effects in SM and MSSM Higgs boson production via gluon fusion at the LHC. For scalar Higgs boson production the results have been analyzed at the $\mathrm{N}^{3} \mathrm{LL}$ level, while pseudoscalar Higgs boson production can only be extended to the NNLL level with the present state-of-the-art calculations. We have implemented top mass effects up to the NLL level by using the full mass dependence of the finite part of the virtual corrections according to [20]. The bottom and charm loops, however, have been treated at fixed NLO rigorously since there are additional nonresummed double logarithmic contributions that have a large impact on the size of their contribution. We have discussed an alternative extension of the formerly used approach for the inclusion of collinear gluon effects at $\mathcal{O}(1 / N)$ in Mellin space to the subleading logarithmic level as a conjecture. ${ }^{7}$ We have been able to reproduce the leading and subleading $\mathrm{N}^{3} \mathrm{LO}$ terms at $\mathcal{O}(1 / N)$ which is a nontrivial cross-check of our method. The impact of resummation effects on the total cross sections reaches a size of a few percent beyond the fixed-order calculations, while the effect of including top mass effects and subleading collinear logarithms in the resummation ranges at the per-mille level or below.

\section{ACKNOWLEDGMENTS}

We are indebted to C. Anastasiou, J. Blümlein, D. de Florian, M. Grazzini, S. Moch, J. Rojo, R. Thorne, A. Vogt and J. Zurita for useful discussions and helpful clarifications. We are grateful to $\mathrm{R}$. Thorne for providing us with an updated version of the MSTW08 PDFs. This work is supported in part by the Research Executive Agency (REA) of the European Union under Grant No. PITN-GA-2012316704 (Higgstools) and a Forschungskredit by Zürich University.

\footnotetext{
${ }^{7}$ The recently developed next-to-eikonal approach [67] may provide a basis for a factorization proof of logarithmic $\mathcal{O}(1 / N)$ terms.
} 
[1] S. L. Glashow, Partial symmetries of weak interactions, Nucl. Phys. 22, 579 (1961); S. Weinberg, A Model of Leptons, Phys. Rev. Lett. 19, 1264 (1967); A. Salam, Weak and electromagnetic interactions, Conf. Proc. C 680519, 367 (1968).

[2] G. Aad et al. (ATLAS Collaboration), Observation of a new particle in the search for the Standard Model Higgs boson with the ATLAS detector at the LHC, Phys. Lett. B 716, 1 (2012); S. Chatrchyan et al. (CMS Collaboration), Observation of a new boson at a mass of $125 \mathrm{GeV}$ with the CMS experiment at the LHC, Phys. Lett. B 716, 30 (2012).

[3] P. W. Higgs, Broken symmetries, massless particles and gauge fields, Phys. Lett. 12, 132 (1964); Broken Symmetries and the Masses of Gauge Bosons, Phys. Rev. Lett. 13, 508 (1964); Spontaneous symmetry breakdown without massless bosons, Phys. Rev. 145, 1156 (1966); F. Englert and R. Brout, Broken Symmetry and the Mass of Gauge Vector Mesons, Phys. Rev. Lett. 13, 321 (1964); G. S. Guralnik, C. R. Hagen, and T. W. Kibble, Global Conservation Laws and Massless Particles, Phys. Rev. Lett. 13, 585 (1964).

[4] CMS Collaboration, Combination of standard model Higgs boson searches and measurements of the properties of the new boson with a mass near $125 \mathrm{GeV}$, Report No. CMS-PAS-HIG-13-005; ATLAS Collaboration, Combined coupling measurements of the Higgs-like boson with the ATLAS detector using up to $25 \mathrm{fb}^{-1}$ of protonproton collision data, Report No. ATLAS-CONF-2013-034.

[5] G. Aad et al. (ATLAS Collaboration), Evidence for the spin-0 nature of the Higgs boson using ATLAS data, Phys. Lett. B 726, 120 (2013); V. Khachatryan et al. (CMS Collaboration), Constraints on the spin-parity and anomalous HVV couplings of the Higgs boson in proton collisions at 7 and 8 TeV, Phys. Rev. D 92, 012004 (2015).

[6] J. Alcaraz et al. (ALEPH and DELPHI and L3 and OPAL and LEP Electroweak Working Group Collaborations), Precision electroweak measurements and constraints on the Standard Model, arXiv:0712.0929.

[7] H. M. Georgi, S. L. Glashow, M. E. Machacek, and D. V. Nanopoulos, Higgs Bosons from Two Gluon Annihilation in Proton Proton Collisions, Phys. Rev. Lett. 40, 692 (1978).

[8] A. Djouadi, M. Spira, and P. M. Zerwas, Production of Higgs bosons in proton colliders: QCD corrections, Phys. Lett. B 264, 440 (1991); S. Dawson, Radiative corrections to Higgs boson production, Nucl. Phys. B359, 283 (1991); S. Dawson and R. Kauffman, QCD corrections to Higgs boson production: Nonleading terms in the heavy quark limit, Phys. Rev. D 49, 2298 (1994).

[9] M. Spira, A. Djouadi, D. Graudenz, and P. M. Zerwas, Higgs boson production at the LHC, Nucl. Phys. B453, 17 (1995).

[10] D. Graudenz, M. Spira, and P. M. Zerwas, QCD Corrections to Higgs Boson Production at Proton Proton Colliders, Phys. Rev. Lett. 70, 1372 (1993); R. Harlander and P. Kant, Higgs production and decay: Analytic results at next-toleading order QCD, J. High Energy Phys. 12 (2005) 015; C. Anastasiou, S. Bucherer, and Z. Kunszt, HPro: A NLO Monte-Carlo for Higgs production via gluon fusion with finite heavy quark masses, J. High Energy Phys. 10 (2009) 068 .
[11] C. Anastasiou, S. Beerli, S. Bucherer, A. Daleo, and Z. Kunszt, Two-loop amplitudes and master integrals for the production of a Higgs boson via a massive quark and a scalar-quark loop, J. High Energy Phys. 01 (2007) 082; U. Aglietti, R. Bonciani, G. Degrassi, and A. Vicini, Analytic results for virtual QCD corrections to Higgs production and decay, J. High Energy Phys. 01 (2007) 021.

[12] M. Krämer, E. Laenen, and M. Spira, Soft gluon radiation in Higgs boson production at the LHC, Nucl. Phys. B511, 523 (1998); M. Spira, Higgs boson production at the LHC, arXiv:hep-ph/9703355.

[13] J. R. Ellis, M. K. Gaillard, and D. V. Nanopoulos, A phenomenological profile of the Higgs boson, Nucl. Phys. B106, 292 (1976); M. A. Shifman, A. I. Vainshtein, M. B. Voloshin, and V.I. Zakharov, Low-energy theorems for Higgs boson couplings to photons, Yad. Fiz. 30, 1368 (1979) [Sov. J. Nucl. Phys. 30, 711 (1979)]; B. A. Kniehl and M. Spira, Low-energy theorems in Higgs physics, Z. Phys. C 69, 77 (1995).

[14] R. V. Harlander and W. B. Kilgore, Soft and virtual corrections to $p \vec{p} H+x$ at NNLO, Phys. Rev. D 64, 013015 (2001); Next-to-Next-to-Leading Order Higgs Production at Hadron Colliders, Phys. Rev. Lett. 88, 201801 (2002); C. Anastasiou and K. Melnikov, Higgs boson production at hadron colliders in NNLO QCD, Nucl. Phys. B646, 220 (2002); S. Marzani, R. D. Ball, V. Del Duca, S. Forte, and A. Vicini, Higgs production via gluon-gluon fusion with finite top mass beyond next-to-leading order, Nucl. Phys. B800, 127 (2008).

[15] S. Catani, D. de Florian, and M. Grazzini, Higgs production in hadron collisions: Soft and virtual QCD corrections at NNLO, J. High Energy Phys. 05 (2001) 025.

[16] V. Ravindran, J. Smith, and W. L. van Neerven, NNLO corrections to the total cross section for Higgs boson production in hadron hadron collisions, Nucl. Phys. B665, 325 (2003).

[17] R. V. Harlander and K. J. Ozeren, Top mass effects in Higgs production at next-to-next-to-leading order QCD: Virtual corrections, Phys. Lett. B 679, 467 (2009); Finite top mass effects for hadronic Higgs production at next-to-next-toleading order, J. High Energy Phys. 11 (2009) 088; A. Pak, M. Rogal, and M. Steinhauser, Virtual three-loop corrections to Higgs boson production in gluon fusion for finite top quark mass, Phys. Lett. B 679, 473 (2009); Finite top quark mass effects in NNLO Higgs boson production at LHC, J. High Energy Phys. 02 (2010) 025.

[18] S. Catani, D. de Florian, M. Grazzini, and P. Nason, Softgluon resummation for Higgs boson production at hadron colliders, J. High Energy Phys. 07 (2003) 028.

[19] S. Moch and A. Vogt, Higher-order soft corrections to lepton pair and Higgs boson production, Phys. Lett. B 631, 48 (2005); V. Ravindran, On Sudakov and soft resummations in QCD, Nucl. Phys. B746, 58 (2006); Higher-order threshold effects to inclusive processes in QCD, Nucl. Phys. B752, 173 (2006); A. Idilbi, X. d. Ji, J. P. Ma, and F. Yuan, Threshold resummation for Higgs production in effective field theory, Phys. Rev. D 73, 077501 (2006); V. Ahrens, T. Becher, M. Neubert, and L. L. Yang, Renormalizationgroup improved prediction for Higgs production at hadron colliders, Eur. Phys. J. C 62, 333 (2009); D. de Florian and 
M. Grazzini, Higgs production through gluon fusion: Updated cross sections at the Tevatron and the LHC, Phys. Lett. B 674, 291 (2009).

[20] D. de Florian and M. Grazzini, Higgs production at the LHC: Updated cross sections at $\sqrt{s}=8 \mathrm{TeV}$, Phys. Lett. B 718, 117 (2012).

[21] T. Gehrmann, M. Jaquier, E.W. N. Glover, and A. Koukoutsakis, Two-loop QCD corrections to the helicity amplitudes for $H \rightarrow 3$ partons, J. High Energy Phys. 02 (2012) 056; C. Anastasiou, C. Duhr, F. Dulat, and B. Mistlberger, Soft triple-real radiation for Higgs production at N3LO, J. High Energy Phys. 07 (2013) 003; C. Anastasiou, C. Duhr, F. Dulat, F. Herzog, and B. Mistlberger, Real-virtual contributions to the inclusive Higgs cross-section at $\mathrm{N}^{3} \mathrm{LO}$, J. High Energy Phys. 12 (2013) 088; W. B. Kilgore, One-loop single-real-emission contributions to $p p \rightarrow H+X$ at next-to-next-to-next-toleading order, Phys. Rev. D 89, 073008 (2014); Y. Li, A. von Manteuffel, R. M. Schabinger, and H. X. Zhu, $\mathrm{N}^{3} \mathrm{LO}$ Higgs boson and Drell-Yan production at threshold: The one-loop two-emission contribution, Phys. Rev. D 90, 053006 (2014); C. Anastasiou, C. Duhr, F. Dulat, E. Furlan, T. Gehrmann, F. Herzog, and B. Mistlberger, Higgs boson gluon-fusion production beyond threshold in $\mathrm{N}^{3} \mathrm{LO}$ QCD, J. High Energy Phys. 03 (2015) 091.

[22] C. Anastasiou, C. Duhr, F. Dulat, F. Herzog, and B. Mistlberger, Higgs Boson Gluon-Fusion Production in QCD at Three Loops, Phys. Rev. Lett. 114, 212001 (2015).

[23] C. Anastasiou, C. Duhr, F. Dulat, E. Furlan, F. Herzog, and B. Mistlberger, Soft expansion of double-real-virtual corrections to Higgs production at $\mathrm{N}^{3} \mathrm{LO}$, J. High Energy Phys. 08 (2015) 051.

[24] D. de Florian, J. Mazzitelli, S. Moch, and A. Vogt, Approximate $\mathrm{N}^{3} \mathrm{LO}$ Higgs-boson production cross section using physical-kernel constraints, J. High Energy Phys. 10 (2014) 176.

[25] M. Bonvini and S. Marzani, Resummed Higgs cross section at $N^{3}$ LL, J. High Energy Phys. 09 (2014) 007; M. Bonvini and L. Rottoli, Three loop soft function for $\mathrm{N}^{3} \mathrm{LL}^{\prime}$ gluon fusion Higgs production in soft-collinear effective theory, Phys. Rev. D 91, 051301 (2015).

[26] S. Catani, L. Cieri, D. de Florian, G. Ferrera, and M. Grazzini, Threshold resummation at $\mathrm{N}^{3} \mathrm{LL}$ accuracy and soft-virtual cross sections at $\mathrm{N}^{3} \mathrm{LO}$, Nucl. Phys. B888, 75 (2014).

[27] A. Djouadi and P. Gambino, Leading Electroweak Correction to Higgs Boson Production at Proton Colliders, Phys. Rev. Lett. 73, 2528 (1994); K. G. Chetyrkin, B. A. Kniehl, and M. Steinhauser, Virtual Top Quark Effects on the $\mathrm{H} \rightarrow \mathrm{b}$ Anti-b Decay at Next-to-Leading Order in QCD, Phys. Rev. Lett. 78, 594 (1997); Three loop $O\left(\alpha_{s}^{2} G_{F} M_{t}^{2}\right)$ corrections to hadronic Higgs decays, Nucl. Phys. B490, 19 (1997); U. Aglietti, R. Bonciani, G. Degrassi, and A. Vicini, Two loop light fermion contribution to Higgs production and decays, Phys. Lett. B 595, 432 (2004); Two-loop electroweak corrections to Higgs production in protonproton collisions, arXiv:hep-ph/0610033; G. Degrassi and F. Maltoni, Two-loop electroweak corrections to Higgs production at hadron colliders, Phys. Lett. B 600, 255 (2004); S. Actis, G. Passarino, C. Sturm, and S. Uccirati,
NLO electroweak corrections to Higgs boson production at hadron colliders, Phys. Lett. B 670, 12 (2008); S. Actis, G. Passarino, C. Sturm, and S. Uccirati, NNLO computational techniques: The cases $\mathrm{H} \rightarrow \gamma \gamma$ and $\mathrm{H} \rightarrow \mathrm{gg}$, Nucl. Phys. B811, 182 (2009).

[28] C. Anastasiou, R. Boughezal, and F. Petriello, Mixed QCDelectroweak corrections to Higgs boson production in gluon fusion, J. High Energy Phys. 04 (2009) 003.

[29] P. Fayet, Supergauge invariant extension of the Higgs mechanism and a model for the electron and its neutrino, Nucl. Phys. B90, 104 (1975); Supersymmetry and weak, electromagnetic and strong interactions, Phys. Lett. 64B, 159 (1976); Spontaneously broken supersymmetric theories of weak, electromagnetic and strong interactions, Phys. Lett. 69B, 489 (1977); S. Dimopoulos and H. Georgi, Softly broken supersymmetry and SU(5), Nucl. Phys. B193, 150 (1981); N. Sakai, Naturalness in supersymmetric guts, Z. Phys. C 11, 153 (1981); K. Inoue, A. Kakuto, H. Komatsu, and S. Takeshita, Low-energy parameters and particle masses in a supersymmetric grand unified model, Prog. Theor. Phys. 67, 1889 (1982); Aspects of grand unified models with softly broken supersymmetry, Prog. Theor. Phys. 68, 927 (1982); Prog. Theor. Phys. 70, 330(E) (1983); Renormalization of supersymmetry breaking parameters revisited, Prog. Theor. Phys. 71, 413 (1984).

[30] See e.g. G. Degrassi, S. Heinemeyer, W. Hollik, P. Slavich, and G. Weiglein, Towards high-precision predictions for the MSSM Higgs sector, Eur. Phys. J. C 28, 133 (2003).

[31] S. P. Martin, Three-loop corrections to the lightest Higgs scalar boson mass in supersymmetry, Phys. Rev. D 75, 055005 (2007); R. V. Harlander, P. Kant, L. Mihaila, and M. Steinhauser, Higgs Boson Mass in Supersymmetry to Three Loops, Phys. Rev. Lett. 100, 191602 (2008); Phys. Rev. Lett. 101, 039901 (2008); P. Kant, R. V. Harlander, L. Mihaila, and M. Steinhauser, Light MSSM Higgs boson mass to three-loop accuracy, J. High Energy Phys. 08 (2010) 104.

[32] S. Heinemeyer, O. Stål, and G. Weiglein, Interpreting the LHC Higgs search results in the MSSM, Phys. Lett. B 710, 201 (2012); R. Benbrik, M. Gomez Bock, S. Heinemeyer, O. Stål, G. Weiglein, and L. Zeune, Confronting the MSSM and the NMSSM with the discovery of a signal in the two photon channel at the LHC, Eur. Phys. J. C 72, 2171 (2012); A. Bottino, N. Fornengo, and S. Scopel, Phenomenology of light neutralinos in view of recent results at the CERN Large Hadron Collider, Phys. Rev. D 85, 095013 (2012); M. Drees, A supersymmetric explanation of the excess of Higgs-like events at the LHC and at LEP, Phys. Rev. D 86, 115018 (2012); P. Bechtle, S. Heinemeyer, O. Stål, T. Stefaniak, G. Weiglein, and L. Zeune, MSSM interpretations of the LHC discovery: Light or heavy Higgs?, Eur. Phys. J. C 73, 2354 (2013).

[33] CMS Collaboration, Search for charged Higgs bosons with the $\mathrm{H}+$ to tau nu decay channel in the fully hadronic final state at $\sqrt{s}=8 \mathrm{TeV}$, Report No. CMS-PAS-HIG-14-020; G. Aad et al. (ATLAS Collaboration), Search for charged Higgs bosons decaying via $H^{ \pm} \rightarrow \tau^{ \pm} \nu$ in fully hadronic final states using $p p$ collision data at $\sqrt{s}=8 \mathrm{TeV}$ with the ATLAS detector, J. High Energy Phys. 03 (2015) 088.

[34] See e.g. M. Spira, QCD effects in Higgs physics, Fortschr. Phys. 46, 203 (1998); A. Djouadi, The anatomy 
of electro-weak symmetry breaking. II. The Higgs bosons in the minimal supersymmetric model, Phys. Rep. 459, 1 (2008).

[35] R. P. Kauffman and W. Schaffer, QCD corrections to production of Higgs pseudoscalars, Phys. Rev. D 49, 551 (1994); A. Djouadi, M. Spira, and P. M. Zerwas, Two photon decay widths of Higgs particles, Phys. Lett. B 311, 255 (1993).

[36] M. Spira, A. Djouadi, D. Graudenz, and P. M. Zerwas, SUSY Higgs production at proton colliders, Phys. Lett. B 318, 347 (1993).

[37] R. V. Harlander and W. B. Kilgore, Production of a pseudoscalar Higgs boson at hadron colliders at next-to-next-to leading order, J. High Energy Phys. 10 (2002) 017; C. Anastasiou and K. Melnikov, Pseudoscalar Higgs boson production at hadron colliders in NNLO QCD, Phys. Rev. D 67, 037501 (2003).

[38] D. de Florian and J. Zurita, Soft-gluon resummation for pseudoscalar Higgs boson production at hadron colliders, Phys. Lett. B 659, 813 (2008).

[39] S. Dawson, A. Djouadi, and M. Spira, QCD Corrections to SUSY Higgs Production: The Role of Squark Loops, Phys. Rev. Lett. 77, 16 (1996).

[40] M. Mühlleitner and M. Spira, Higgs boson production via gluon fusion: Squark loops at NLO QCD, Nucl. Phys. B790, 1 (2008); R. Bonciani, G. Degrassi, and A. Vicini, Scalar particle contribution to Higgs production via gluon fusion at NLO, J. High Energy Phys. 11 (2007) 095.

[41] R. V. Harlander and M. Steinhauser, Hadronic Higgs production and decay in supersymmetry at next-to-leading order, Phys. Lett. B 574, 258 (2003); Effects of SUSY-QCD in hadronic Higgs production at next-to-next-to-leading order, Phys. Rev. D 68, 111701 (2003); Supersymmetric Higgs production in gluon fusion at next-to-leading order, J. High Energy Phys. 09 (2004) 066; R. V. Harlander and F. Hofmann, Pseudo-scalar Higgs production at next-toleading order SUSY-QCD, J. High Energy Phys. 03 (2006) 050; G. Degrassi and P. Slavich, On the NLO QCD corrections to Higgs production and decay in the MSSM, Nucl. Phys. B805, 267 (2008); NLO QCD bottom corrections to Higgs boson production in the MSSM, J. High Energy Phys. 11 (2010) 044; G. Degrassi, S. Di Vita, and P. Slavich, NLO QCD corrections to pseudoscalar Higgs production in the MSSM, J. High Energy Phys. 08 (2011) 128; On the NLO QCD corrections to the production of the heaviest neutral Higgs scalar in the MSSM, Eur. Phys. J. C 72, 2032 (2012).

[42] T. Appelquist and J. Carazzone, Infrared singularities and massive fields, Phys. Rev. D 11, 2856 (1975).

[43] M. Mühlleitner, H. Rzehak, and M. Spira, MSSM Higgs boson production via gluon fusion: The large gluino mass limit, J. High Energy Phys. 04 (2009) 023.

[44] C. Anastasiou, S. Beerli, and A. Daleo, The Two-Loop QCD Amplitude gg $\rightarrow \mathrm{h}, \mathrm{H}$ in the Minimal Supersymmetric Standard Model, Phys. Rev. Lett. 100, 241806 (2008); M. Mühlleitner, H. Rzehak, and M. Spira, SUSY-QCD corrections to MSSM Higgs boson production via gluon fusion, Proc. Sci. RADCOR2009 (2010) 043 [arXiv:1001.3214]; MSSM Higgs boson production via gluon fusion, DESYPROC-2010-01.
[45] G. Altarelli and G. Parisi, Asymptotic freedom in parton language, Nucl. Phys. B126, 298 (1977).

[46] G. F. Sterman, Summation of large corrections to short distance hadronic cross-sections, Nucl. Phys. B281, 310 (1987); S. Catani and L. Trentadue, Resummation of the QCD perturbative series for hard processes, Nucl. Phys. B327, 323 (1989); Comment on QCD exponentiation at large x, Nucl. Phys. B353, 183 (1991).

[47] A. Vogt, Next-to-next-to-leading logarithmic threshold resummation for deep inelastic scattering and the Drell-Yan process, Phys. Lett. B 497, 228 (2001).

[48] S. Moch, J. A. M. Vermaseren, and A. Vogt, Higher-order corrections in threshold resummation, Nucl. Phys. B726, 317 (2005); E. Laenen and L. Magnea, Threshold resummation for electroweak annihilation from DIS data, Phys. Lett. B 632, 270 (2006).

[49] T. Schmidt, Ph.D. thesis, Zürich University, 2015.

[50] S. Moch and A. Vogt, On non-singlet physical evolution kernels and large-x coefficient functions in perturbative QCD, J. High Energy Phys. 11 (2009) 099.

[51] M. I. Kotsky and O. I. Yakovlev, On the resummation of double logarithms in the process $H \rightarrow \gamma \gamma$, Phys. Lett. B 418, 335 (1998); R. Akhoury, H. Wang, and O. I. Yakovlev, On the resummation of large QCD logarithms in $H \rightarrow \gamma \gamma$ decay, Phys. Rev. D 64, 113008 (2001).

[52] S. Forte, A. Isgrò, and G. Vita, Do we need $\mathrm{N}^{3} \mathrm{LO}$ parton distributions?, Phys. Lett. B 731, 136 (2014).

[53] M. Bonvini, S. Marzani, J. Rojo, L. Rottoli, M. Ubiali, R. D. Ball, V. Bertone, S. Carrazza, and N. P. Hartland, Parton distributions with threshold resummation, J. High Energy Phys. 09 (2015) 191.

[54] M. Spira, HIGLU: A program for the calculation of the total Higgs production cross-section at hadron colliders via gluon fusion including QCD corrections, arXiv:hep-ph/9510347; HIGLU and HDECAY: Programs for Higgs boson production at the LHC and Higgs boson decay widths, Nucl. Instrum. Methods Phys. Res., Sect. A 389, 357 (1997); R. Harlander, M. Mühlleitner, J. Rathsman, M. Spira, and O. Stål, Interim recommendations for the evaluation of Higgs production cross sections and branching ratios at the LHC in the two-Higgs-doublet model, arXiv:1312.5571.

[55] R. V. Harlander, S. Liebler, and H. Mantler, SusHi: A program for the calculation of Higgs production in gluon fusion and bottom-quark annihilation in the Standard Model and the MSSM, Comput. Phys. Commun. 184, 1605 (2013).

[56] A. Vogt, Efficient evolution of unpolarized and polarized parton distributions with QCD-PEGASUS, Comput. Phys. Commun. 170, 65 (2005).

[57] A. D. Martin, W. J. Stirling, R. S. Thorne, and G. Watt, Parton distributions for the LHC, Eur. Phys. J. C 63, 189 (2009).

[58] S. Catani, M. L. Mangano, P. Nason, and L. Trentadue, The top cross-section in hadronic collisions, Phys. Lett. B 378, 329 (1996); S. Catani, M. L. Mangano, P. Nason, and L. Trentadue, The resummation of soft gluons in hadronic collisions, Nucl. Phys. B478, 273 (1996).

[59] A. Kulesza, G. F. Sterman, and W. Vogelsang, Joint resummation in electroweak boson production, Phys. Rev. D 66, 014011 (2002); Joint resummation for Higgs production, Phys. Rev. D 69, 014012 (2004). 
[60] S. Dittmaier et al. (LHC Higgs Cross Section Working Group Collaboration), Handbook of LHC Higgs cross sections: 1. Inclusive observables, arXiv:1101.0593.

[61] J. Gao, M. Guzzi, J. Huston, H.-L. Lai, Z. Li, P. Nadolsky, J. Pumplin, D. Stump, and C.-P. Yuan, CT10 next-to-nextto-leading order global analysis of QCD, Phys. Rev. D 89, 033009 (2014).

[62] R. D. Ball et al., Parton distributions with LHC data, Nucl. Phys. B867, 244 (2013).

[63] S. Alekhin, J. Blumlein, and S. Moch, The ABM parton distributions tuned to LHC data, Phys. Rev. D 89, 054028 (2014).

[64] V. Radescu (H1 and ZEUS Collaborations), Combination and QCD analysis of the HERA inclusive cross sections, Proc. Sci. ICHEP2010 (2010) 168; A. M. Cooper-Sarkar (ZEUS and H1 Collaborations), PDF fits at HERA, Proc. Sci. EPS HEP2011 (2011) 320 [arXiv:1112.2107].
[65] M. Carena, S. Heinemeyer, O. Stl, C. E. M. Wagner, and G. Weiglein, MSSM Higgs boson searches at the LHC: Benchmark scenarios after the discovery of a Higgs-like particle, Eur. Phys. J. C 73, 2552 (2013).

[66] M. S. Carena, M. Quiros, and C. E. M. Wagner, Effective potential methods and the Higgs mass spectrum in the MSSM, Nucl. Phys. B461, 407 (1996).

[67] E. Laenen, L. Magnea, and G. Stavenga, On next-to-eikonal corrections to threshold resummation for the Drell-Yan and DIS cross sections, Phys. Lett. B 669, 173 (2008); E. Laenen, L. Magnea, G. Stavenga, and C. D. White, Next-to-eikonal corrections to soft gluon radiation: A diagrammatic approach, J. High Energy Phys. 01 (2011) 141; D. Bonocore, E. Laenen, L. Magnea, S. Melville, L. Vernazza, and C. D. White, A factorization approach to next-to-leading-power threshold logarithms, J. High Energy Phys. 06 (2015) 008. 